\section{Erfolgreiches Immuntherapie-Präparat}

eit seiner Einführung wurden mittlerweile mehr als 1 Million Allergiepatienten mit Allergovit ${ }^{\circledR}$ hyposensibilisiert. In mehreren Untersuchungen konnte gezeigt werden, dass das Präparat eine sichere und klinisch wirksame Therapieoption für $\mathrm{Pa}$ tienten mit pollenbedingter allergischer Rhinitis ist.

Eine doppelblinde, placebokontrollierte Studie beispielsweise bestätigte die klinische Effektivität und Verträglichkeit der subkutanen Immuntherapie (SCIT) mit
Routineanwendung in der Praxis übertragen lassen [Martin E et al. Allergy 2009; 64: 345]. Die Beobachtungsstudie erfasste von 2001 bis 2005 die Daten von 2.931 Patienten mit IgE-assoziierter Allergie und klinischen Symptomen wie Rhinitis, Konjunktivitis und/oder Asthma in 221 Praxen. Bereits nach einem präsaisonalen Behandlungszyklus mit dem Allergoid wurden anhand einer visuellen Analogskala bezüglich des Patientenbefindens positive Effekte registriert: Bei 86,3\% aller Patienten kam

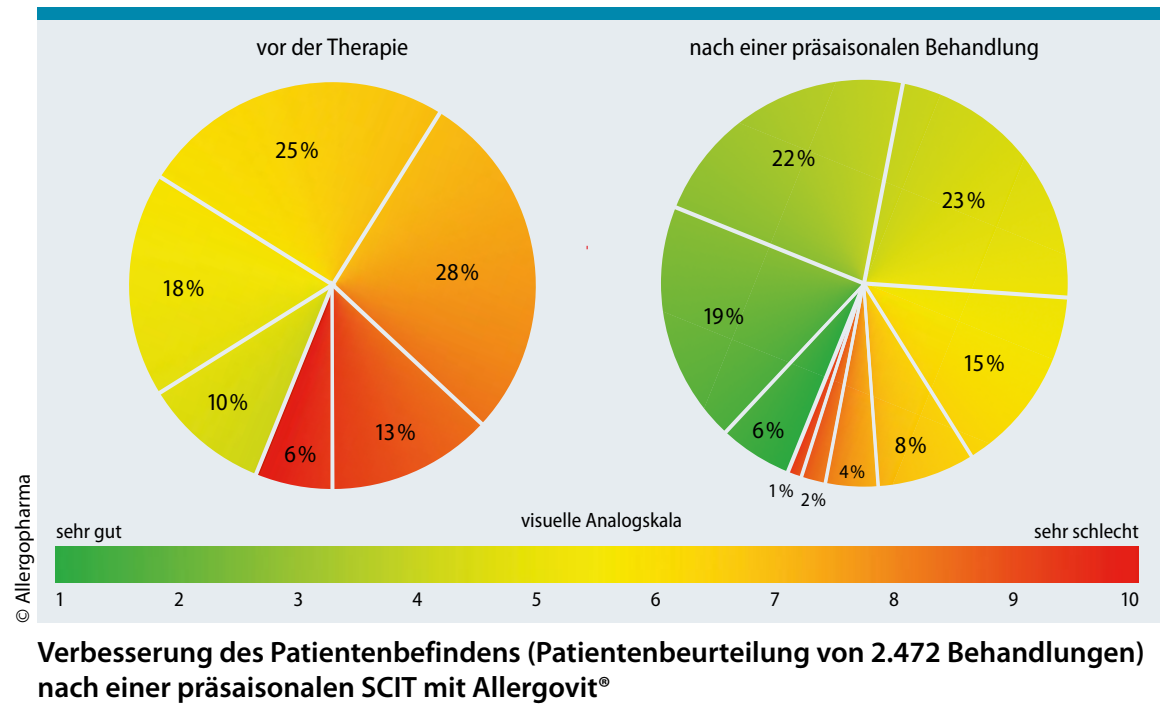

Allergovit ${ }^{\circledR}$ [Corrigan C et al. Allergy 2005; 60 801-7]. An der Studie, die zwei Jahre lang lief, nahmen 154 erwachsene Gräserpollenallergiker teil. Bereits in der Behandlungsphase kam es zu einer kontinuierlichen und signifikanten Verbesserung des Symptom-Medikations-Scores unter dem 6-Gräserpollen-Allergoid. Die Untersuchung zeigt zudem die Sicherheit des Präparats in der täglichen Praxis: Nur bei 5,5\% aller Patienten gab es eine lokale Injektionsreaktion (Placebo: 0,24\%).

Darüber hinaus gibt es Hinweise aus anderen Studien auf eine über den Behandlungszeitraum hinausgehende Langzeitwirksamkeit nach Ende der SCIT mit Allergovit $^{\circledast}$ Gräser bei Kindern und Erwachsenen [Eng PA et al. Allergy 2002; 57: 306-11- Narkus A. et al. Allergy 2009; 64: 346 - Kettner J et al. Allergo J 2009; 18: 50].

Eine nicht interventionelle Untersuchung kommt zu dem Schluss, dass sich die klinischen Studiendaten auch auf die es durch die Behandlung zu einer Verbesserung um mindestens zwei Punkte auf der visuellen Analogskala. Gleichzeitig konnten $68,7 \%$ der Patienten den Gebrauch zusätzlicher Medikamente verringern. Nur bei $8,2 \%$ der insgesamt verabreichten 27.109 Injektionen kam es zu unerwünschten Wirkungen, wobei es sich bei $98,8 \%$ um geringfügige lokale Reaktionen handelte. 95\% der Patienten wollten die Therapie weiterführen.

Das vorteilhafte Nutzen-Risiko-Profil von Allergovit ${ }^{\circledast}$ hat auch das Paul-EhrlichInstitut bestätigt. Im Sicherheitsreport des Instituts heißt es: „Das hohe Sicherheitsprofil von Allergovit ${ }^{\circledR}$ wird bestätigt durch die niedrige Intensität und Inzidenz der beobachteten Nebenwirkungen. Die Nutzen-Risiko-Einschätzung des Allergoids erweist sich als ebenso vorteilhaft wie das Sicherheitsprofil.“

Nach Informationen von Allergopharma

\section{Fixierbinde ohne Naturkautschuk}

Eine neue latexfreie, bi-elastische, kohäsive Fixierbinde (Mollelast ${ }^{\circledR}$ haft latexfrei) kommt Ärzten und Patienten mit Latexallergie zugute. Eine nicht allergene, synthetische Mikrobeschichtung sorgt dabei für den guten kohäsiven Effekt. Außerdem besitzt Mollelast ${ }^{\circledR}$ haft latexfrei eine besondere Bi-Elastizität in Quer- und Längsrichtung, wodurch sich die Binde sowohl an konische als auch an runde Körperpatien anpasst. Das sorgt für einen sicheren Halt auch in der häufig bewegten Gelenkregion. Ein weiterer Vorteil der latexfreien Fixierbinde sind die Webkanten. Diese fixieren die einzelnen Fäden und verhindern ein Ausfransen der Binde. Dabei besitzt das besonders dünn gewirkte Material eine hohe Luft- und Wasserdampfdurchlässigkeit, wodurch Wärmestaus und Hautrötungen sowie Schwitzen verhindert werden.

Nach Informationen von Lohmann \& Rauscher

\section{App „Allergy Track“}

Die neue App „Allergy Track" hilft Menschen mit allergischer Rhinitis dabei, ihre Allergie zu dokumentieren und bei Veränderungen so früh wie möglich einen Arzt aufzusuchen. Aus Sicht von Prof. Dr. Margitta Worm, Berlin, profitieren sowohl Allergiker als auch Ärzte von dem Service: „Viele Allergiker sind nicht ausreichend vertraut mit Atemwegsallergien und ihren Auswirkungen. Eine App wie Allergy Track hilft dabei, wertvolle Informationen über die eigene Allergie, z. B. den Verlauf der Symptome, zu sammeln und kann den Austausch wichtiger Informationen - beispielsweise das Beschwerdebild in der Pollensaison - zwischen Arzt und Patient verbessern." Mit wenigen Eingaben können Nutzer von Allergy Track die Intensität ihrer Symptome und andere Auswirkungen der Allergie auf das tägliche Leben bestimmen. Eine Tagebuchfunktion ermöglicht es, den Verlauf der Allergie zu verfolgen, die gesammelten Daten auszudrucken und z. B. einem Arzt vorzulegen. Die integrierte Suchfunktion hilft dabei, den nächstgelegenen Allergologen zu finden. Die App ist kostenlos für iPhone, Smartphone, PC oder MAC unter der Adresse www.stallergenes.de erhältlich.

Nach Informationen von Stallergenes 